\title{
Innovations for Global Health Equity: Beyond Open Access towards Open Data
}

\section{By Emma Veitch PhD, Virginia Barbour MB BChir DPhil}

Addressing inequity in health information is a crucial step in reducing worldwide disparities in health outcomes, and a necessary prerequisite for achieving the Millennium Development Goals.[1] The current lack of global equity in this area applies to widely diverse resources, including peer-reviewed journal articles, reference material such as drug formularies and teaching material, and even basic health systems and administrative information, such as birth and death registration data.

The advent of Open Access (OA) journal publication addresses one component of this inequity, represented and advocated by the Public Library of Science (PLoS) and many other publishers. Compared to "traditional" subscription-based journals, OA publishing removes barriers to access based on price (i.e. subscription fees) and permission (copyright restrictions on reuse). Key is a liberal license, allowing article users to reproduce, distribute, and reuse the work worldwide with proper attribution.

Many funders of biomedical and population health research anticipate that the effects of such wider access will be to increase the impact of their grantees' research on future studies, policy and practice, and thus now mandate free or open access to its results. Examples include the Wellcome Trust in the UK and the National Institutes of Health in the USA.

$\mathrm{OA}$ is an essential prerequisite to developing other tools permitting a more comprehensive approach to broaden accessibility to critical health information. These tools include creation of OA repositories, such as PubMed Central (http://www.ncbi. nlm.nih.gov/pmc/), which provides perpetual archiving for OA literature. Ultimately, the intention is that through linking of such archives to other public data sources, automated dataand text-mining will be able to deliver novel biomedical and population health insights.

Yet OA publication and repositories are only a small part of effectively addressing the inequities in distribution and access to results of primary research. In fact, evidence suggests that reliance on primary research journals as the exclusive tool for timely dissemination of data relevant to health policy does not fulfill all the needs of the scientific community, especially in times of emergency. An evaluation of epidemiological studies on the 2003 SARS outbreak found that only a very small proportion of published studies were actually submitted while the epidemic was underway, and were not available to inform control policies until long after the epidemic's end.[2] In the study, the researchers found that at the beginning of the epidemic, papers were mostly published in "high-impact" journals, but as the epidemic waned, they were more likely to appear in lower impact journals. For these later papers, multiple rounds of review, initially by higher impact journals, may have contributed to delays in publication. The study's authors proposed that standardized tools be developed for rapid preparation and dissemination of such research reports, perhaps using mechanisms other than peer-reviewed journals.
Finally, addressing the global health information gap obliges us to look beyond primary research literature to issues relating to underlying data. Recently, eight global health agencies (Bill \& Melinda Gates Foundation; Global Alliance for Vaccines and Immunisation; Global Fund to Fight AIDS, Tuberculosis and Malaria; Human Development Network; Joint United Nations Program on HIVIAIDS; United Nations Children's Fund; United Nations Population Fund, and the World Health Organization) declared that efforts of their organizations and of national health systems are severely limited by glaring data gaps.[3] These often include areas fundamental to developing policy for health systems strengthening, such as output of household survey studies; surveillance data essential for determining disease prevalence; birth and death registrations; census data; and health systems documentation, including financial and human resources information. Importantly, the eight agencies together committed to scaling up funding for data collection, monitoring, evaluation, and operational research. Just as significant, they also made a public commitment to develop "a set of specific principles around data sharing by our organizations within two years", and announced that they would provide funding streams dedicated to enabling data sharing.

One model of such data sharing is the World Bank's new Open Data Initiative (http://data.worldbank.org/), offering free access to over 2000 development indicators from its over 200 member governments worldwide. An open programming interface allows researchers to freely interrogate World Bank data with their own applications.

Before such data sharing can become the norm in health research, however, a number of challenges need to be addressed. These include thorny issues such as confidentiality for patientspecific information; data ownership and permanence; and methods of conferring academic credit.

Clearly, there is a long way to go in fulfilling the potential current technology offers for free and widespread dissemination of health information. The barriers are not only technical, but also political and societal, from the desires of researchers to publish in "high impact" journals to the paucity, for many countries, of meaningful data on the burden of ill-health. Enabling open access to the data we generate is a crucial first start. $M$ -

1. Godlee F, Pakenham-Walsh N, Ncayiyana D, Cohen B, Packer A. Can we achieve health information for all by 2015? Lancet [Internet]. 2004 Jul 17-23 [cited 2010 June 14];364(9430):295-300. Available from: http://www.thelancet.com/ journals/lancet/article/PIIS0140-6736\%2804\%2916681-6/fulltext DOI:10.1016/ S0140-6736(04)16681-6

2. Xing W, Hejblum G, Leung GM, Valleron AJ. Anatomy of the Epidemiological Literature on the 2003 SARS Outbreaks in Hong Kong and Toronto: A Time-Stratified Review. PLoS Med [Internet]. 2010 May 4 [cited 2010 June 14];7(5):e1000272. Available from: http://www.plosmedicine.org/article/info\%3 Adoi\%2F10.1371\%2Fjournal.pmed.1000272

3. Chan M, Kazatchkine M, Lob-Levyt J, Obaid T, Schweizer J, Sidibe M, et al. Meeting the Demand for Results and Accountability: A Call for Action on Health Data from Eight Global Health Agencies. PLoS Med [Internet]. 2010 Jan 26 [cited 2010 June 14];7(1):e1000223. Available from: http://www.plosmedicine.org/article/info\%3Adoi\%2F10.1371\%2Fjournal.pmed.1000223

Disclosures: The authors are employed by the Public Library of Science, an open-access publisher. 